\title{
Coupled-channel effects in elastic scattering and near-barrier fusion induced by weakly bound nuclei and exotic halo nuclei
}

\author{
C. Beck, ${ }^{1, *}$ N. Keeley, ${ }^{2, \dagger}$ and A. Diaz-Torres ${ }^{3}$ \\ ${ }^{1}$ Institut Pluridisciplinaire Hubert Curien, UMR7178, IN2P3-CNRS et Université Louis Pasteur (Strasbourg I), 23 rue du Loess - BP28, \\ F-67037 Strasbourg Cedex 2, France \\ ${ }^{2}$ DSMIDAPNIA/SPhN CEA Saclay, Orme des Merisiers, F-91191 Gif-sur-Yvette Cedex, France \\ ${ }^{3}$ Department of Nuclear Physics, Research School of Physical Sciences and Engineering, The Australian National University, \\ Canberra ACT 0200, Australia
}

(Received 26 January 2007; published 8 May 2007)

\begin{abstract}
The influence on fusion of coupling to the breakup process is investigated for reactions where at least one of the colliding nuclei has a sufficiently low binding energy for breakup to become an important process. Elastic scattering, excitation functions for sub- and near-barrier fusion cross sections, and breakup yields are analyzed for ${ }^{6,7} \mathrm{Li}+{ }^{59} \mathrm{Co}$. Continuum-discretized coupled-channels (CDCC) calculations describe well the data at and above the barrier. Elastic scattering with ${ }^{6} \mathrm{Li}$ (as compared to ${ }^{7} \mathrm{Li}$ ) indicates the significant role of breakup for weakly bound projectiles. A study of ${ }^{4,6} \mathrm{He}$ induced fusion reactions with a three-body $\mathrm{CDCC}$ method for the ${ }^{6} \mathrm{He}$ halo nucleus is presented. The relative importance of breakup and bound-state structure effects on total fusion is discussed.
\end{abstract}

DOI: 10.1103/PhysRevC.75.054605

PACS number(s): 25.70.Bc, 25.70.Jj, 25.70.Mn, 25.70.Gh

\section{INTRODUCTION}

In reactions induced by light weakly bound nuclei, the influence on the fusion process of couplings both to collective degrees of freedom and to breakup (BU) or transfer (TR) channels is a key point for a deeper understanding of few-body systems in quantum dynamics [1,2]. Due to the very weak binding energies of halo nuclei, such as ${ }^{6} \mathrm{He}$ or ${ }^{11} \mathrm{Be}[3-7]$, a diffuse cloud of neutrons should lead to enhanced tunneling probabilities below the Coulomb barrier, where the neutron tail which extends well beyond the compact nuclear core provides a conduit by which the matter distributions of the target and projectile may overlap at longer range than for the core. In the vicinity of the Coulomb barrier and below, enhanced fusion yields with ${ }^{11} \mathrm{Be}$ were predicted [3] but not confirmed experimentally for ${ }^{11,10} \mathrm{Be}+{ }^{209} \mathrm{Bi}$ reactions [8]. For ${ }^{6} \mathrm{He}$, there is some evidence for enhanced fusion probability compared to the ${ }^{4} \mathrm{He}$ core at deep sub-barrier energies in the ${ }^{6} \mathrm{He}+{ }^{206} \mathrm{~Pb}$ system [10] (a same observation has been recently shown for the ${ }^{197} \mathrm{Au}$ target [11]). A model of "sequential fusion" [9] where the fusion enhancement effect was assumed to be due to the gain in energy from a rearrangement of the ${ }^{6} \mathrm{He}$ valence neutrons (due to the positive Q-values for one- and two-neutron TR) was able to predict successfully the data before the experiment. However, most other recent experimental studies involving ${ }^{6} \mathrm{He}$ radioactive ion beams (RIB) $[4,12-18]$ indicate that the halo of the ${ }^{6} \mathrm{He}$ nucleus does not enhance the fusion probability, but illustrate the preponderant role of one- and two-neutron TR in ${ }^{6} \mathrm{He}$ induced reactions $[14,17]$. Hence, the question of a real new effect with RIBs and with weakly bound

\footnotetext{
*Corresponding author. Email address: christian.beck@ires.in 2p3.fr

${ }^{\dagger}$ Permanent address: Department of Nuclear Reactions, The Andrzej Sołtan Institute for Nuclear Studies, Hoża 69, PL-00681, Warsaw, Poland.
}

stable beams such as ${ }^{6} \mathrm{Li},{ }^{7} \mathrm{Li}$, and ${ }^{9} \mathrm{Be}$ remains open [5-7]: namely the occurrence of nonconventional transfer/stripping processes with large cross sections most likely originating from the small binding energy of the projectile as well as the specific role of the BU process have still to be clearly determined. More exclusive measurements will be necessary to disentangle the different components.

Since coupling between channels is known to enhance the fusion cross section at sub-barrier energies [3,19], coupledchannels (CC) effects have often been taken into account in the theoretical description of the quantum tunneling in fusing systems [1-3,6]. A large number of experimental results have been interpreted adequately well within the framework of CC calculations $[1,2,6,7]$. However, in the case of loosely bound (and/or halo) systems the situation is more complicated since the BU and TR channels may induce strong couplings to an infinite number of unbound states in the continuum of the projectile. A possible treatment of the problem is to reduce it to a finite number of states. This is often achieved by discretizing in energy the continuum of the weakly bound nucleus such that the resulting set of coupled equations may be solved in the conventional manner. This is the so-called method of continuum-discretized coupled channels (CDCC) [20-34]. With the recent advent of new RIB facilities [5-7], it is now necessary to extend the CDCC formalism to allow for four-body BU and/or excitation of the "core" nucleus (the question of the treatment of TR channels is also still open). Studies have been initiated in this direction by several groups [35-40] to investigate reactions induced by an exotic "Borromean" $\left({ }^{6} \mathrm{He}\right)$ nucleus, which is known to have a strong dipole excitation mode [41], and the single neutron halo nucleus ${ }^{11} \mathrm{Be}$, where collective excitation of the ${ }^{10} \mathrm{Be}$ core is expected to be important.

In this work we present CDCC calculations describing simultaneously the elastic scattering and limited available BU data for the weakly bound stable nuclei ${ }^{6} \mathrm{Li}$ and ${ }^{7} \mathrm{Li}$ interacting with the medium-mass ${ }^{59} \mathrm{Co}$ target and separate calculations 
for the total fusion (TF) of these nuclei with ${ }^{59} \mathrm{Co}$ and for ${ }^{6} \mathrm{He}$ with ${ }^{59} \mathrm{Co}$ and ${ }^{63,65} \mathrm{Cu}$. Preliminary reports of this work have been presented elsewhere in conference proceedings [42,43]. A description of the CDCC calculations is given in Sec. II. The CDCC results and corresponding comparisons with available experimental elastic scattering, BU, and TF cross sections are discussed in Sec. III. Section IV provides a brief summary as well as suggesting future directions for experimental and theoretical investigations.

\section{CONTINUUM-DISCRETIZED COUPLED-CHANNEL CALCULATIONS}

The fully quantum-mechanical CDCC method, first proposed in the early 1970s by Rawitscher [20] to study the effect of deuteron breakup on elastic scattering, has been widely applied by the Kyushu group [21-24] to study heavy-ion collisions induced by light weakly bound nuclei. CDCC calculations have been successful in the past in describing the scattering of deuterons [22,24] and ${ }^{6,7} \mathrm{Li}[23]$ on different targets. The standard three-body CDCC method has also been applied to reactions involving halo nuclei, e.g., ${ }^{8} \mathrm{~B}$ [25] and ${ }^{6} \mathrm{He}$ [28]. Diaz-Torres and Thompson [26] have used a novel method based on the CDCC formalism to perform a fully quantum-mechanical calculation of TF of the halo nucleus ${ }^{11} \mathrm{Be}$ with a ${ }^{208} \mathrm{~Pb}$ target, later refined and applied to the $\mathrm{TF}$ of ${ }^{6,7} \mathrm{Li}$ [29]. A recent study of the ${ }^{6} \mathrm{He}+{ }^{209} \mathrm{Bi}$ reaction by means of a three-body CDCC model [30] found much larger absorption cross sections than those extracted from optical model (OM) fits to the elastic scattering data [13], a problem that is confirmed by more realistic four-body CDCC calculations [38] that describe the data well. However, the problem with the simple three-body CDCC model for ${ }^{6} \mathrm{He}$ breakup has been traced to the $E 1$ coupling strength; when these couplings are reduced by $50 \%$ good agreement with the data is obtained [34].

In the present work we employ the standard three-body CDCC model to analyze the elastic scattering and BU in the ${ }^{6,7} \mathrm{Li}+{ }^{59} \mathrm{Co}$ systems. Our choice of systems was mainly influenced by the fact that we have already carried out extended CDCC calculations for both the ${ }^{6} \mathrm{Li}+{ }^{59} \mathrm{Co}$ and ${ }^{7} \mathrm{Li}+{ }^{59} \mathrm{Co}$ TF reactions [29] which experimental data were previously published in [44-47] and elastic scattering data are also available for these systems [48]. We also present calculations of the TF of ${ }^{6} \mathrm{He}+{ }^{59} \mathrm{Co}$ and ${ }^{6} \mathrm{He}+{ }^{63,65} \mathrm{Cu}$ using the simplified two-body ${ }^{4} \mathrm{He}+{ }^{2} n$ dineutron model of ${ }^{6} \mathrm{He}$ with the CDCC fusion model of [29]. For these medium-mass targets Coulomb breakup effects should be smaller than with the heavy ${ }^{209} \mathrm{Bi}$ target, so we have chosen not to apply the $50 \%$ reduction of the $E 1$ coupling strength of [34] here. All calculations were carried out using the code FRESCO [49].

\section{A. CDCC calculations of ${ }^{7} \mathrm{Li}+{ }^{59} \mathrm{Co}$ and ${ }^{6} \mathrm{Li}+{ }^{59} \mathrm{Co}$ elastic scattering}

The CDCC calculations applied to the elastic scattering were carried out assuming an $\alpha+d(t)$ cluster structure for ${ }^{6} \mathrm{Li}\left({ }^{7} \mathrm{Li}\right)$. The $\alpha+d$ and $\alpha+t$ binding potentials were taken from Refs. [50] and [51], respectively. However, the radius of the $\alpha+d$ binding potential was increased to $R=2.56 \mathrm{fm}$ to obtain better agreement with the measured $B\left(E 2 ; 1^{+} \rightarrow 3^{+}\right)$. The $\alpha+d$ wave functions calculated using this potential yield a $B(E 2)$ of $24.0 e^{2} \mathrm{fm}^{4}$, in excellent agreement with the measured value of $25.6 \pm 2.0 e^{2} \mathrm{fm}^{4}$ [52]. The calculations were otherwise similar to those described in [27,30]. The continuum model space was limited to cluster relative angular momentum values of $L=0,1,2$, and 3 for both $\mathrm{Li}$ isotopes, sufficient or more than sufficient (for the case of ${ }^{6} \mathrm{Li}$ ) to provide convergent results for the elastic scattering and BU. The $\alpha+d(t)$ continuum was discretized into a series of bins in momentum space of width $\Delta k=0.2 \mathrm{fm}^{-1}$ with $0.0 \leqslant k \leqslant 1.0 \mathrm{fm}^{-1}$, where $\hbar k$ denotes the momentum of the $\alpha+d(t)$ relative motion. All couplings, including continuumcontinuum couplings, up to multipolarity $\lambda=3$ were included. For the calculations at incident ${ }^{6,7} \mathrm{Li}$ energies of $18 \mathrm{MeV}$ the continuum space was truncated at $k_{\max }=0.8 \mathrm{fm}^{-1}$. Test calculations at 30 and $26 \mathrm{MeV}$ using this truncation gave identical results to those with $k_{\max }=1.0 \mathrm{fm}^{-1}$. At $12 \mathrm{MeV}$ truncation of the continuum at $k_{\max }=0.6 \mathrm{fm}^{-1}$ was found to be sufficient. Interaction and coupling potentials were generated using the cluster-folding procedure.

The $\alpha+{ }^{59} \mathrm{Co}$ and $d(t)+{ }^{59} \mathrm{Co}$ potentials required as input for this procedure were obtained as follows. The $\alpha+{ }^{59} \mathrm{Co}$ potentials were obtained by adjusting the real and imaginary well depths of the global $\alpha$ potential of Avrigeanu et al. [53] to match the $24.7 \mathrm{MeV} \alpha+{ }^{59} \mathrm{Co}$ elastic scattering data of McFadden and Satchler [54], resulting in normalizations $N_{\mathrm{R}}=0.67$ and $N_{\mathrm{I}}=2.52$ for the real and imaginary depths, respectively. These normalizations were then applied to the global potential calculated at the appropriate energies, there being no suitable data available to fix this input more precisely. The $d(t)+{ }^{59}$ Co potentials were the unmodified global potentials of Perey and Perey [55] and Becchetti and Greenlees [56], respectively, there being no suitable scattering data available in the literature.

The real and imaginary well depths of the cluster-folded ${ }^{6,7} \mathrm{Li}+{ }^{59} \mathrm{Co}$ potentials (including the coupling potentials) were adjusted to obtain the optimum description of the elastic scattering data. The CDCC calculations are compared with the elastic scattering data of [48] in Figs. 1 and 2 for ${ }^{7} \mathrm{Li}+{ }^{59} \mathrm{Co}$ and ${ }^{6} \mathrm{Li}+{ }^{59} \mathrm{Co}$, respectively.

The curves show the results of calculations with (solid lines) and without (dashed lines) ${ }^{6,7} \mathrm{Li} \rightarrow \alpha+d, t$ breakup couplings. It is worth noting that the dashed line of Fig. 1 has been calculated with reorientation of ${ }^{7} \mathrm{Li}$ g.s. The effect of $\mathrm{BU}$ on the elastic scattering, stronger for ${ }^{6} \mathrm{Li}$ as expected, is illustrated in Figs. 1 and 2 by the difference between the one-channel (i.e., no coupling) calculations and the full CDCC results. A similar effect was also observed for the ${ }^{6,7} \mathrm{Li}+{ }^{65} \mathrm{Cu}$ elastic scattering [57] at $25 \mathrm{MeV}$ incident energy.

OM fits to the data were also carried out to obtain total reaction cross sections. The starting point for the $\mathrm{OM}$ fits to the ${ }^{6} \mathrm{Li}+{ }^{59} \mathrm{Co}$ data was the potential of Fulmer et al. [58] for ${ }^{6} \mathrm{Li}+{ }^{59} \mathrm{Co}$ elastic scattering at an incident energy of $88 \mathrm{MeV}$. For the ${ }^{7} \mathrm{Li}+{ }^{59} \mathrm{Co}$ data we used the global ${ }^{7} \mathrm{Li}$ optical potential of Cook [59]. The real and imaginary potential depths and the 

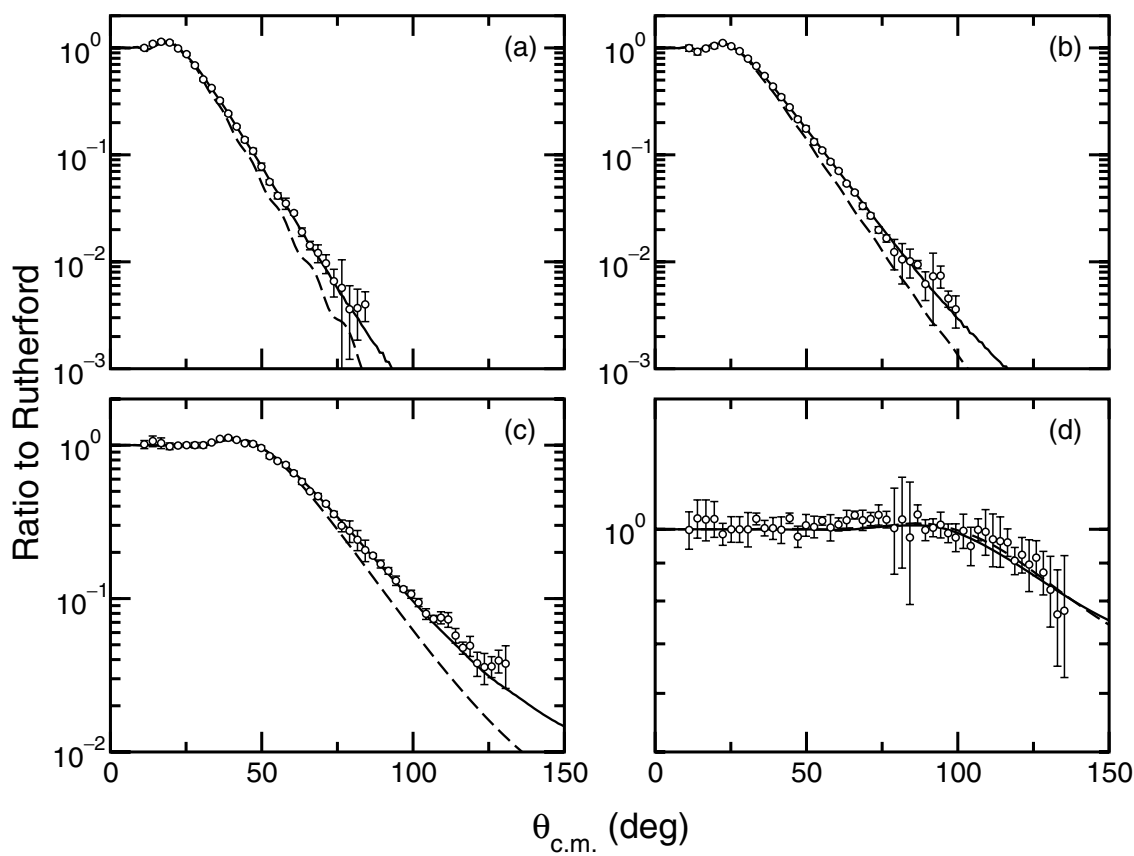

FIG. 1. Ratios of the elastic scattering cross sections to the Rutherford cross sections as a function of c.m. angle for the ${ }^{7} \mathrm{Li}+{ }^{59} \mathrm{Co}$ system [48]. The curves correspond to calculations with (solid lines) and without (dashed lines) ${ }^{7} \mathrm{Li} \rightarrow \alpha+t$ breakup couplings to the continuum for incident ${ }^{7} \mathrm{Li}$ energies of (a) $30 \mathrm{MeV}$, (b) $26 \mathrm{MeV}$, (c) $18 \mathrm{MeV}$, and (d) $12 \mathrm{MeV}$. imaginary diffuseness were searched on in both cases, all other parameters being held fixed. The resulting best fit parameters are given in Tables I and II.

The total reaction cross sections obtained from the CDCC calculations are in good agreement with those obtained from the OM fits, see Tables III and IV, except at $12 \mathrm{MeV}$ where the relatively poor precision of the data means that both the $\mathrm{OM}$ potential parameters and the total reaction cross sections are poorly determined.

We would particularly like to point out that for both systems the calculated total BU cross sections are negligible fractions of the total reaction cross sections, either calculated with $\mathrm{CDCC}$ or obtained from OM fits, which latter may be regarded as "experimental" values.

For ${ }^{6} \mathrm{Li}+{ }^{59} \mathrm{Co}$ the calculated $\mathrm{BU}$ cross sections are between $3.7-9.7 \%$ of the calculated total reaction cross sections, while for ${ }^{7} \mathrm{Li}+{ }^{59} \mathrm{Co}$ the corresponding values are between $0.6-1.0 \%$. The lower values for ${ }^{7} \mathrm{Li}$ may be ascribed partly to the higher breakup threshold ( $S_{\alpha}=2.47 \mathrm{MeV}$ compared to $1.47 \mathrm{MeV}$ for ${ }^{6} \mathrm{Li}$ ), partly to the presence of a bound excited state (the 0.478 $\mathrm{MeV} 1 / 2^{-}$) and strong ground state reorientation coupling, absent in ${ }^{6} \mathrm{Li}$.

We may verify in part our conclusions concerning the small contribution of $\mathrm{BU}$ to the total reaction cross section, as data for
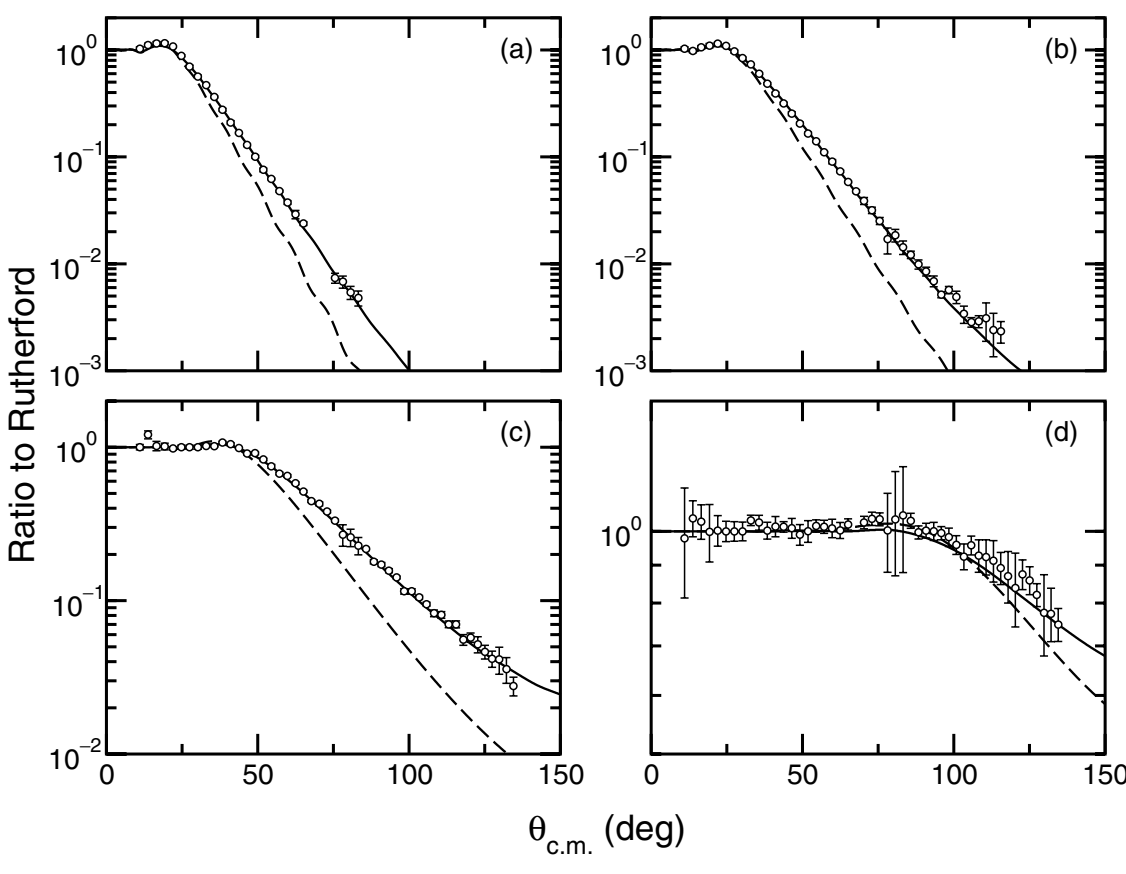

10

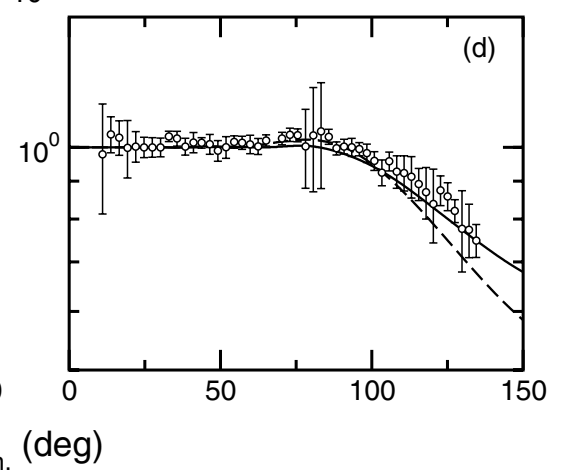

FIG. 2. Ratios of the elastic scattering cross sections to the Rutherford cross sections as a function of c.m. angle for the ${ }^{6} \mathrm{Li}+{ }^{59} \mathrm{Co}$ system [48]. The curves correspond to calculations with (solid lines) and without (dashed lines) ${ }^{6} \mathrm{Li} \rightarrow \alpha+d$ breakup couplings to the continuum for incident ${ }^{6} \mathrm{Li}$ energies of (a) $30 \mathrm{MeV}$, (b) $26 \mathrm{MeV}$, (c) $18 \mathrm{MeV}$, and (d) $12 \mathrm{MeV}$. 
TABLE I. OM fits to the ${ }^{7} \mathrm{Li}+{ }^{59} \mathrm{Co}$ elastic scattering data.

\begin{tabular}{lccccccc}
\hline \hline$E_{\text {lab }}$ & $\begin{array}{c}V \\
(\mathrm{MeV})\end{array}$ & $\begin{array}{c}r_{V} \\
(\mathrm{fm})\end{array}$ & $\begin{array}{c}a_{V} \\
(\mathrm{fm})\end{array}$ & $\begin{array}{c}W \\
(\mathrm{MeV})\end{array}$ & $\begin{array}{c}r_{W} \\
(\mathrm{fm})\end{array}$ & $\begin{array}{l}a_{W} \\
(\mathrm{fm})\end{array}$ & $\chi^{2} / N$ \\
\hline 30 & 100.0 & 1.286 & 0.853 & 18.8 & 1.739 & 0.7814 & 0.88 \\
26 & 108.7 & 1.286 & 0.853 & 22.7 & 1.739 & 0.8050 & 0.64 \\
18 & 114.5 & 1.286 & 0.853 & 25.2 & 1.739 & 0.7367 & 0.52 \\
12 & 179.1 & 1.286 & 0.853 & 8.91 & 1.739 & 0.6941 & 0.66 \\
\hline \hline
\end{tabular}

the sequential BU of ${ }^{6} \mathrm{Li}$ via the $2.18 \mathrm{MeV}^{+}$excited state are available for a $41 \mathrm{MeV}^{6} \mathrm{Li}$ beam incident on a ${ }^{59} \mathrm{Co}$ target [60]. Sequential BU via this state is the dominant contribution to the total ${ }^{6} \mathrm{Li} \rightarrow \alpha+d$ breakup cross section. There are no elastic scattering data available at this energy, so we adjusted our CDCC calculation to give good agreement with the elastic scattering calculated using the best fit OM potential parameters for the $44 \mathrm{MeV}^{6} \mathrm{Li}+{ }^{54} \mathrm{Fe}$ data of [61], used in [60] as the basis for a DWBA calculation of the "inelastic scattering" to the ${ }^{6} \mathrm{Li}$ $3^{+}$state. We compare our CDCC calculation with the data of [60] in Fig. 3.

The calculated angular distribution is somewhat smaller than the measured one; a renormalization of the latter by a factor of $2 / 3$ would result in good agreement with the calculation. This discrepancy in magnitude is reflected in the integrated cross sections; Bochkarev et al. [60] give a value of $45 \pm 10 \mathrm{mb}$ whereas the CDCC calculation gives a value of $22.5 \mathrm{mb}$. We note that we were unable to reproduce the data with a DWBA calculation using the measured $B(E 2)$ value of $25.6 e^{2} \mathrm{fm}^{4}$ [52] (Bochkarev et al. do not give details of their DWBA calculation) but that good agreement was obtained when we multiplied this value by 1.5 . Thus, it is possible that there is a slight normalization factor error, of the order of $2 / 3$, in the data of [60], in which case our calculation would be in excellent agreement with the data. In any case, even if the CDCC total BU cross sections are too small by a factor of about 1.5, this does not affect the conclusion that $\mathrm{BU}$ contributes negligibly to the total reaction cross section at these near-barrier energies.

In Figs. 4 and 5 we present the integrated total reaction cross sections, total BU cross sections, ${ }^{6} \mathrm{Li} 2.18 \mathrm{MeV} 3^{+}$sequential $\mathrm{BU}$ cross sections and the ${ }^{7} \mathrm{Li}$ ground state reorientation plus $1 / 2^{-}$inelastic excitation cross sections. We also show the fusion cross sections obtained from barrier penetration model (BPM) calculations using the real part of the bare potential plus the "trivially equivalent local potential" derived from the breakup couplings, the latter calculated using the method described in [62]. These quantities are compared with the total

TABLE II. OM fits to the ${ }^{6} \mathrm{Li}+{ }^{59} \mathrm{Co}$ elastic scattering data.

\begin{tabular}{lccccccc}
\hline \hline$E_{\text {lab }}$ & $\begin{array}{c}V \\
(\mathrm{MeV})\end{array}$ & $\begin{array}{c}r_{V} \\
(\mathrm{fm})\end{array}$ & $\begin{array}{c}a_{V} \\
(\mathrm{fm})\end{array}$ & $\begin{array}{c}W \\
(\mathrm{MeV})\end{array}$ & $\begin{array}{c}r_{W} \\
(\mathrm{fm})\end{array}$ & $\begin{array}{l}a_{W} \\
(\mathrm{fm})\end{array}$ & $\chi^{2} / N$ \\
\hline 30 & 66.9 & 1.265 & 0.901 & 13.6 & 1.760 & 0.7632 & 1.27 \\
26 & 75.0 & 1.265 & 0.901 & 16.6 & 1.760 & 0.7675 & 0.66 \\
18 & 71.3 & 1.265 & 0.901 & 21.2 & 1.760 & 0.7905 & 1.27 \\
12 & 113.4 & 1.265 & 0.901 & 16.3 & 1.760 & 0.7114 & 0.41 \\
\hline \hline
\end{tabular}

TABLE III. Total reaction cross sections and integrated BU cross sections obtained from the CDCC calculations for ${ }^{7} \mathrm{Li}+{ }^{59} \mathrm{Co}$. The total reaction cross sections extracted from $\mathrm{OM}$ fits to the elastic scattering data are also given for comparison, along with the measured TF cross sections [44].

\begin{tabular}{lcccc}
\hline \hline$E_{\text {lab }}$ & $\begin{array}{c}\sigma_{R}(\mathrm{OM}) \\
(\mathrm{mb})\end{array}$ & $\begin{array}{c}\sigma_{R}(\mathrm{CDCC}) \\
(\mathrm{mb})\end{array}$ & $\sigma_{\text {bu }}(\mathrm{mb})$ & $\sigma_{\text {fus }}(\mathrm{mb})$ \\
\hline 30 & 1603 & 1610 & 16.6 & - \\
26 & 1547 & 1596 & 12.8 & $1014 \pm 204$ \\
18 & 888 & 876 & 4.67 & $547 \pm 110$ \\
12 & 45.4 & 83.5 & 0.31 & $38 \pm 8$ \\
\hline \hline
\end{tabular}

reaction cross sections obtained from the OM fits to the elastic scattering data and the measured TF cross sections of [44]. While the method used to calculate the fusion cross sections is rather crude, it does appear to have some value as a means of providing a reasonable estimate of the TF cross section (to within about $20 \%$ or so) which may be useful when planning experiments.

It is clear from Figs. 4 and 5 and Tables III and IV that the total reaction cross section is dominated by fusion at these near and above barrier energies (the nominal Coulomb barrier for these systems is equivalent to an incident Li energy of about $14 \mathrm{MeV})$. Due to the rather large error bars on the measured TF cross sections [44] it is not possible to draw definite conclusions, but it is evident from the tables that the sum of TF yields plus BU yields does not exhaust the total reaction cross section except for the data at $12 \mathrm{MeV}$, where the total reaction cross section is less well defined by the elastic scattering data. The discrepancy may be accounted for by inelastic excitation of the target (expected to be relatively unimportant for ${ }^{59} \mathrm{Co}$, which does not exhibit a high degree of collectivity), ground state reorientation plus inelastic excitation of the $0.78 \mathrm{MeV}$ $1 / 2^{-}$state in ${ }^{7} \mathrm{Li}$ and $\mathrm{TR}$ reactions. It should be noted that these other direct reactions make a considerably greater contribution to the total reaction cross section than does BU. The bulk of this remaining cross section is probably due to TR reactions of the $\left({ }^{7} \mathrm{Li},{ }^{6} \mathrm{Li}\right),\left({ }^{7} \mathrm{Li},{ }^{8} \mathrm{Be}\right),\left({ }^{6} \mathrm{Li},{ }^{5} \mathrm{Li}\right)$, etc., type-as may be seen from Fig. 4, even when the cross sections for ground state reorientation and inelastic excitation of the $1 / 2^{-}$state are added to the $\mathrm{TF}$ and $\mathrm{BU}$ cross sections for ${ }^{7} \mathrm{Li}$ the total reaction cross section is far from being exhausted by the sum.

TABLE IV. Total reaction cross sections and integrated BU cross sections obtained from the CDCC calculations for ${ }^{6} \mathrm{Li}+{ }^{59} \mathrm{Co}$. The total reaction cross sections extracted from $\mathrm{OM}$ fits to the elastic scattering data are also given for comparison, along with the measured TF cross sections [44].

\begin{tabular}{lcccc}
\hline \hline$E_{\mathrm{lab}}$ & $\begin{array}{c}\sigma_{R}(\mathrm{OM}) \\
(\mathrm{mb})\end{array}$ & $\begin{array}{c}\sigma_{R}(\mathrm{CDCC}) \\
(\mathrm{mb})\end{array}$ & $\sigma_{\text {bu }}(\mathrm{mb})$ & $\sigma_{\text {fus }}(\mathrm{mb})$ \\
\hline 30 & 1480 & 1581 & 61.4 & - \\
26 & 1401 & 1448 & 55.0 & $988 \pm 199$ \\
18 & 934 & 973 & 34.2 & $467 \pm 94$ \\
12 & 77.2 & 116.0 & 7.46 & $57 \pm 12$ \\
\hline \hline
\end{tabular}




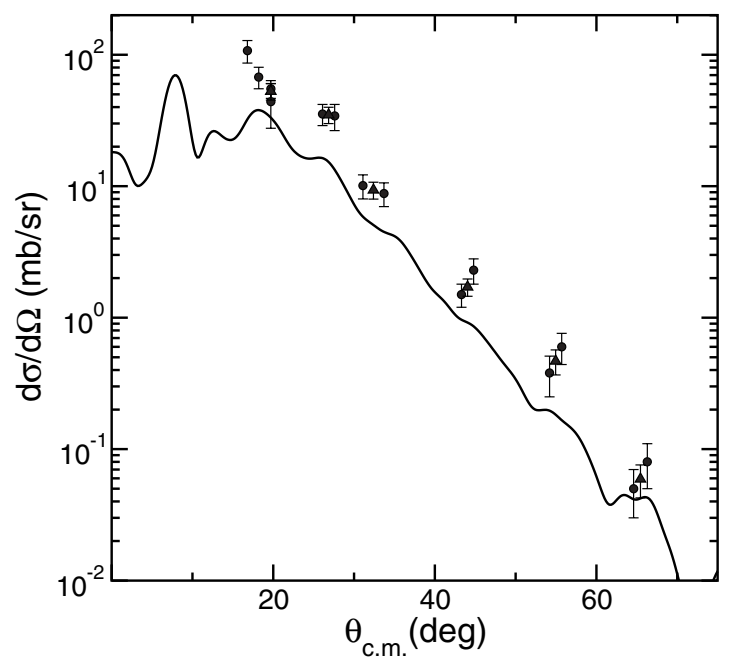

FIG. 3. CDCC calculation for the angular distribution of the ${ }^{6} \mathrm{Li}$ $\rightarrow \alpha+d$ sequential breakup via the $2.18 \mathrm{MeV} 3^{+}$state of ${ }^{6} \mathrm{Li}$ compared to the data of Bochkarev et al. [60] as obtained for the ${ }^{6} \mathrm{Li}+{ }^{59} \mathrm{Co}$ reaction at $41 \mathrm{MeV}$.

Unfortunately, we were unable to confirm our inference by calculating the TR cross sections for ${ }^{59} \mathrm{Co}$, as the density of states in the final nuclei is too high. However, a rough estimate of the contribution due to single nucleon stripping and pickup reactions was attempted through a series of DWBA calculations. Due to their incomplete nature-limitations in the number of states that it was possible to include mean that the resulting cross sections are to be regarded more as lower limits — we give only a brief outline of the calculations here.
The entrance channel optical potentials were taken from Tables I and II as appropriate, while the mass 5 and 6 and mass 7 and 8 exit channel optical potentials were calculated using the ${ }^{6} \mathrm{Li}$ and ${ }^{7} \mathrm{Li}$ global parameters of [59], respectively. The projectile-like overlap spectroscopic factors were taken from [63] and the transferred nucleons were bound in Woods-Saxon wells of radius $r_{0}=1.25 \mathrm{fm}$ and diffuseness $a=0.65 \mathrm{fm}$, plus a spin-orbit component of the same geometry with a fixed depth of $6 \mathrm{MeV}$, the depth of central part being adjusted to give the correct binding energy. The spectroscopic factors and binding potentials for the target-like overlaps were taken from [64-67].

The summed integrated cross sections are plotted on Figs. 4 and 5 as the filled triangles. From these results we may infer that the total single nucleon transfer cross sections are at least as large as the total breakup cross sections for ${ }^{6} \mathrm{Li}$ and rather larger than the total breakup cross sections for ${ }^{7} \mathrm{Li}$ over most of the incident energy range of interest here. Nevertheless, we are still far from being able to account for all the total reaction cross section at the highest energies. Possible candidates for the missing cross section are cluster transfers such as $\left({ }^{6} \mathrm{Li},{ }^{4} \mathrm{He}\right)$ or $\left({ }^{7} \mathrm{Li},{ }^{4} \mathrm{He}\right)$, although the large positive Q-values for these reactions make any meaningful estimate of the cross sections impossible, as little or nothing is known of the structure of the target-like fragments in the kinematically important excitation energy regime.

The real and imaginary parts of the sum of the bare plus dynamic polarization potentials (DPPs) generated by the couplings to BU are presented in Figs. 6 and 7 (filled circles) along with the best $\mathrm{OM}$ fits potentials (open circles) for comparison. The error bars on the best fit OM values were obtained by gridding on the real diffuseness parameter while searching on the imaginary well depth and diffuseness, all other parameters being held fixed at the best fit values. The

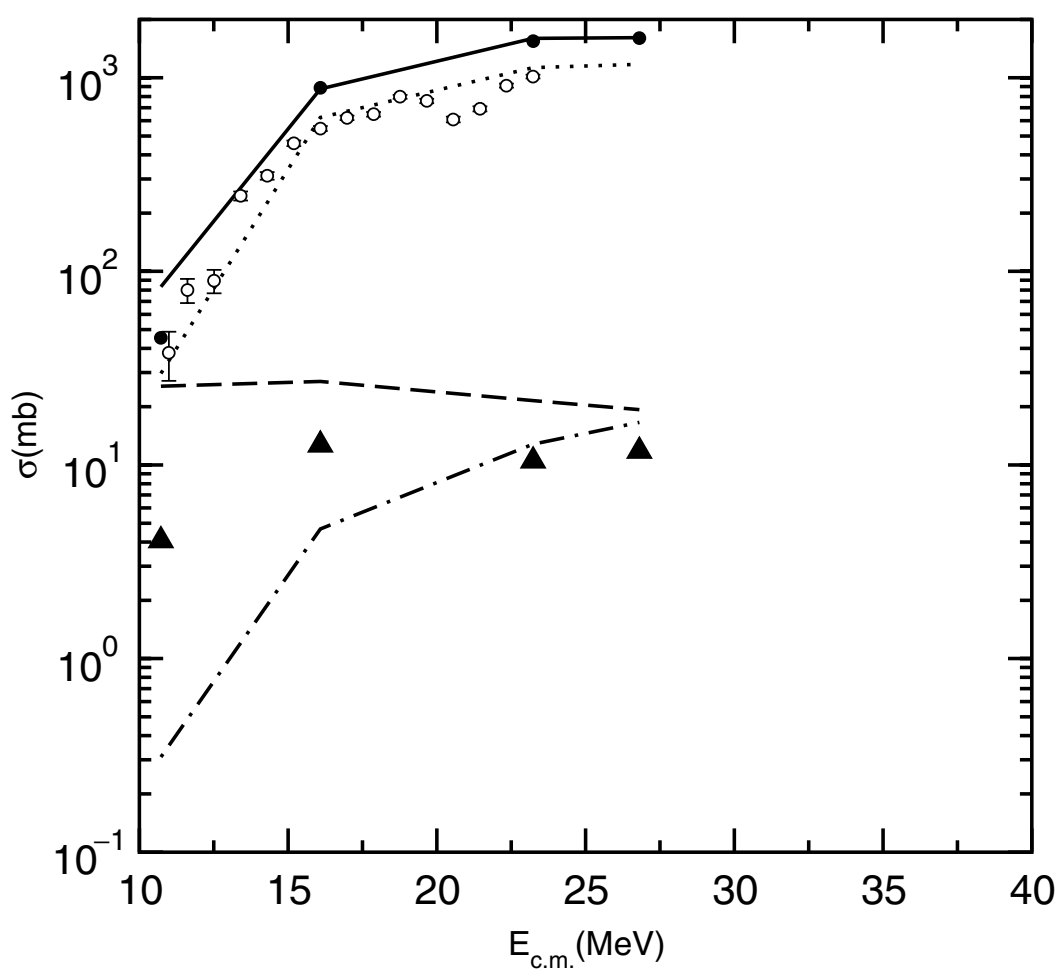

FIG. 4. Total reaction cross sections (solid curve), integrated total BU cross sections (dotdashed curve), integrated ${ }^{7} \mathrm{Li}$ ground state reorientation plus $1 / 2^{-}$inelastic excitation cross sections (dashed curve) and BPM fusion cross sections (dotted curve) obtained from the CDCC calculations for the ${ }^{7} \mathrm{Li}+{ }^{59} \mathrm{Co}$ system. The filled and open circles denote the total reaction cross sections obtained from the best OM fits and the measured TF cross sections [44], respectively. The filled triangles denote the summed DWBA estimates for single nucleon stripping and pickup reactions, see text for details. 


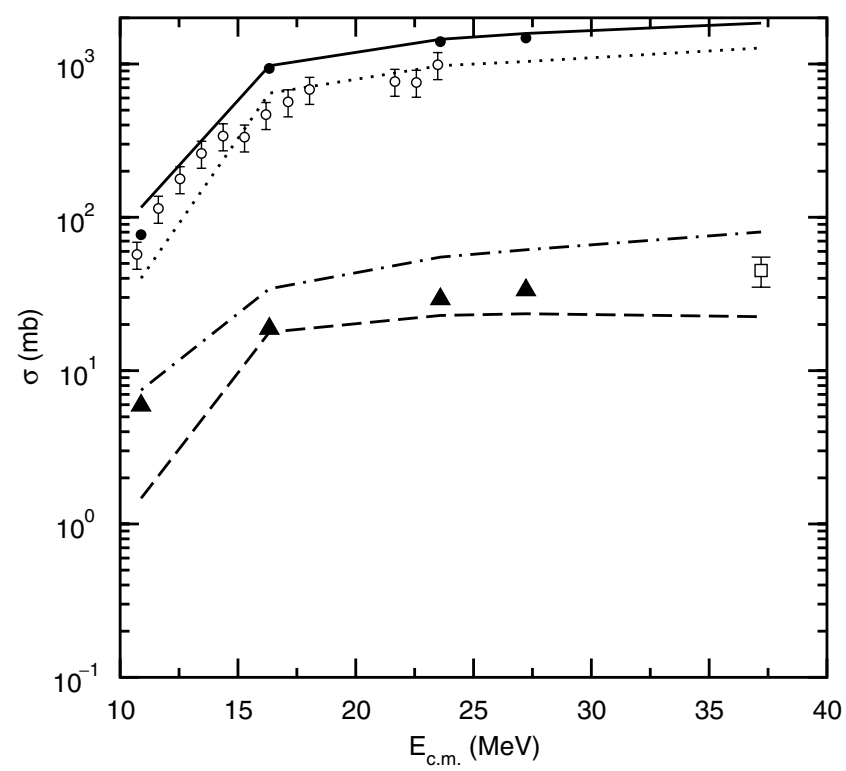

FIG. 5. Total reaction cross sections (solid curve), integrated total BU cross sections (dot-dashed curve), integrated ${ }^{6} \mathrm{Li} 2.18 \mathrm{MeV}^{+}$ sequential BU cross sections (dashed curve) and BPM fusion cross sections (dotted curve) obtained from the CDCC calculations for the ${ }^{6} \mathrm{Li}+{ }^{59} \mathrm{Co}$ system. The filled and open circles denote the total reaction cross sections obtained from the best OM fits and the measured TF cross sections [44], respectively. The open square denotes the ${ }^{6} \mathrm{Li}$ $2.18 \mathrm{MeV}^{+}$sequential BU cross section reported in [60]. The filled triangles denote the summed DWBA estimates for single nucleon stripping and pickup reactions, see text for details.
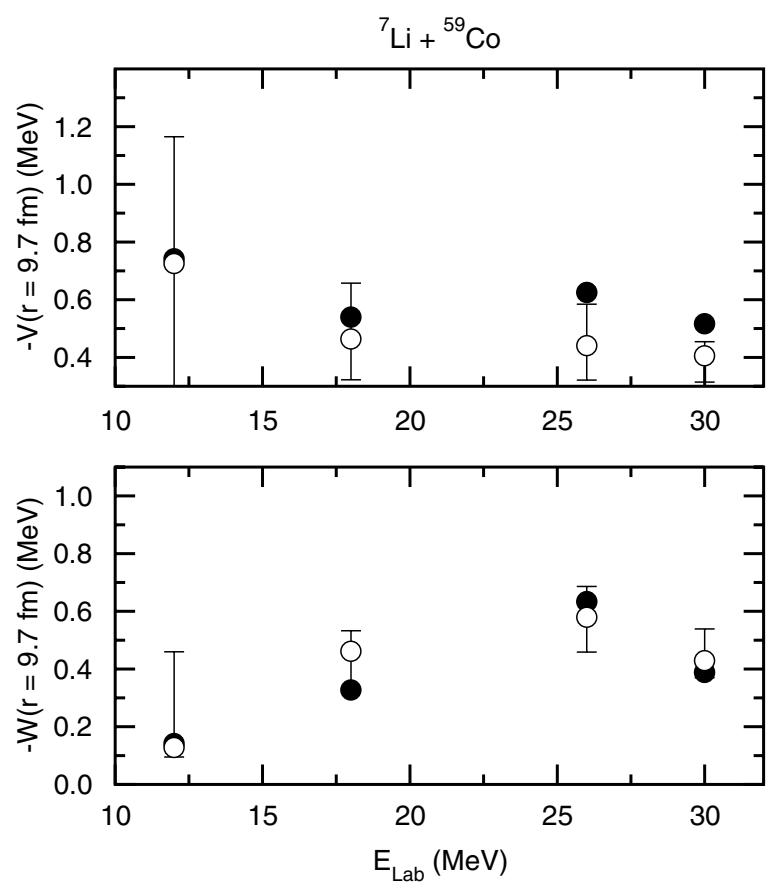

FIG. 6. Energy dependence of the real and imaginary parts of the bare plus DPP potentials as generated by the CDCC calculations (filled circles) and the best $\mathrm{OM}$ fits potentials (open circles) for the ${ }^{7} \mathrm{Li}+{ }^{59} \mathrm{Co}$ system at a radial distance of $r=9.7 \mathrm{fm}$ as discussed in the text.
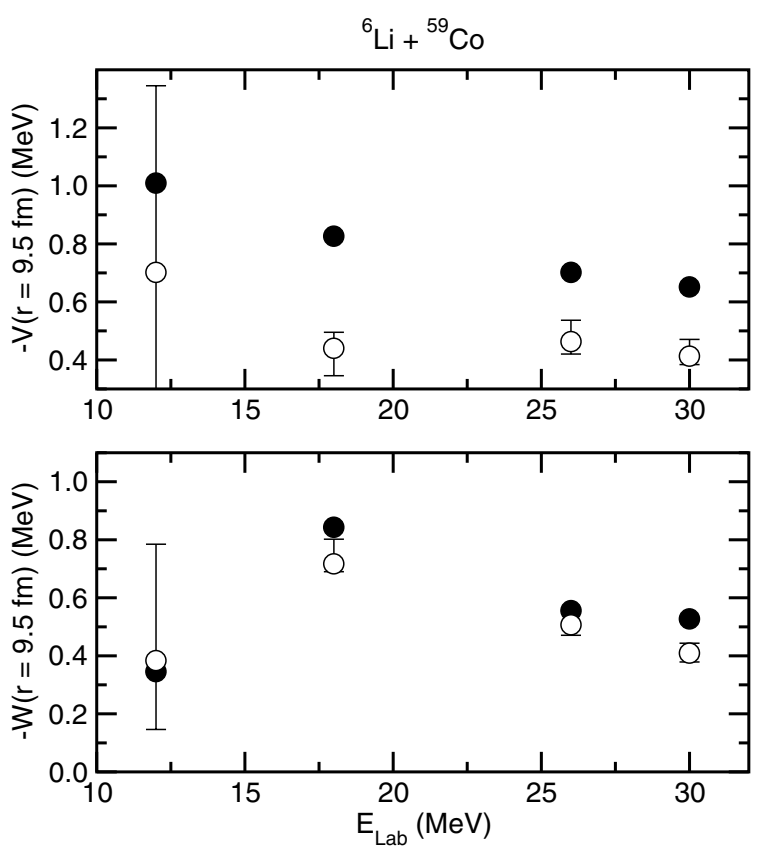

FIG. 7. As for Fig. 6 but for the ${ }^{6} \mathrm{Li}+{ }^{59} \mathrm{Co}$ system at a radial distance of $r=9.5 \mathrm{fm}$.

limits are defined by $\chi^{2} / N$ values of 1.0 (for those data sets where the best fit $\chi^{2} / N$ value is less than 1.0 ) or $15 \%$ larger than the minimum value (for those data sets where the minimum $\chi^{2} / N$ is greater than 1.0).

The potentials are evaluated at radial distances of 9.7 and $9.5 \mathrm{fm}$ for ${ }^{7} \mathrm{Li}$ and ${ }^{6} \mathrm{Li}$, respectively. These values are the mean strong absorption radii obtained from the best $\mathrm{OM}$ fits potentials at 18,26 , and $30 \mathrm{MeV}$ for each system (the results at $12 \mathrm{MeV}$ were omitted due to the large uncertainties in the $\mathrm{OM}$ fits to these data). The difference of $0.2 \mathrm{fm}$ in the "radii of sensitivity" is not significant, as in reality the elastic scattering data probe the nuclear potential over a region of width $\sim 1 \mathrm{fm}$ in the nuclear surface at a given energy rather than at a single radius (which latter, if taken at face value, would violate the uncertainty principle, see, e.g., [68]).

In general, the surface strengths of the "bare plus DPP" potentials are in very good agreement with those of the best OM fits potentials, the exception being the real potentials for the ${ }^{6} \mathrm{Li}+{ }^{59} \mathrm{Co}$ system where the total potentials derived from the CDCC calculations are consistently larger than the OM values. At first sight, one would conclude that the surface potential strengths for both systems exhibit the energy dependence characteristic of the "threshold anomaly", i.e., a rise in the strength of the real part as the incident energy is reduced toward the Coulomb barrier accompanied by a drop in that of the imaginary part. However, this conclusion largely rests on the values at $12 \mathrm{MeV}$ incident energy, and as can be seen from the error bars, the potential strength in the nuclear surface is effectively not determined by the data due to its rather poor precision, a very wide range of values giving equally good fits to the data for both systems. The spread in values for the other energies, while much less than that at $12 \mathrm{MeV}$, is still such that we are unable to draw any concrete conclusions 
concerning the presence or absence of a threshold anomaly (TA) in these systems.

\section{B. CDCC calculations of ${ }^{6} \mathrm{He}+{ }^{59} \mathrm{Co}$ fusion reaction}

Calculations employing the CDCC model for TF of [29] were also carried out to describe the fusion process induced by the "Borromean" nucleus ${ }^{6} \mathrm{He}$ on the same medium-mass target ${ }^{59} \mathrm{Co}$. Firstly, we would like to stress that in these calculations-unlike those for the ${ }^{6,7} \mathrm{Li}$ elastic scattering and $\mathrm{BU}$ reported in the previous section - the imaginary components of the off-diagonal couplings in the transition potentials were neglected, while the diagonal couplings include imaginary parts [29]. Otherwise full continuum couplings were taken into account. We used short-range imaginary potentials for each projectile fragment separately (for example, $\alpha$ and $d+$ target potentials for the case of the ${ }^{6} \mathrm{Li}$ nucleus). This is equivalent to the use of the incoming wave boundary condition in CCFULL calculations [44]; however, only the $\mathrm{TF}$ cross sections can be evaluated with this model. Ideally, one would wish to employ this version of CDCC in a single calculation that attempts to describe the ensemble of the data, TF, BU, TR and elastic scattering. However, we are still some way from being able to include all the necessary direct reaction couplings in a single practicable calculation, at least for systems where fusion data exist (this problem applies equally well to the stable weakly bound nuclei as well as $\left.{ }^{6} \mathrm{He}\right)$.

The calculations were similar to those described in more detail in [29] for ${ }^{6} \mathrm{Li}$, but now applied to the two-neutron halo nucleus ${ }^{6} \mathrm{He}$. The present case is much more complicated since ${ }^{6} \mathrm{He}$ breaks into three fragments $(\alpha+n+n)$ instead of two $(\alpha+d)$, and the CDCC method for two-nucleon halo nuclei has not yet been implemented in FRESCO. Hence a dineutron model is adopted for the ${ }^{6} \mathrm{He}+{ }^{59} \mathrm{Co}$ reaction, i.e., we assume a two-body cluster structure of ${ }^{6} \mathrm{He}={ }^{4} \mathrm{He}+{ }^{2} n$ with an $\alpha$ particle core coupled to a single particle representing a dineutron $\left({ }^{2} n\right)$ like cluster.

As in our previous work [29], the real part of the potentials between the fragments and the ${ }^{59} \mathrm{Co}$ target are those obtained with the global Broglia-Winther Woods-Saxon parametrization $[69,70]$. The numerical values for ${ }^{2} n-{ }^{59} \mathrm{Co}$ and for $\alpha-{ }^{59} \mathrm{Co}$ are $V_{o}=-16.89(-31.14) \mathrm{MeV}, r_{o}=1.09$ (1.127) fm and $a=0.63(0.63)$ fm. For the $\alpha-2 n$ binding potential $\left(0^{+}\right.$g.s. $)$we have used the following Woods-Saxon potential: $V_{o}=-40.796 \mathrm{MeV}, r_{o}=1.896 \mathrm{fm}$ and $a=0.3 \mathrm{fm}$. The g.s. binding potential of the $\alpha$ particle and the dineutron provides a $2 s$ bound state of about $-0.975 \mathrm{MeV}$. The binding potential of the $2^{+}$resonant state also has a Woods-Saxon form with the following parameters: $V_{o}=-35.137 \mathrm{MeV}$, $r_{o}=1.896 \mathrm{fm}, a=0.3 \mathrm{fm}$. With this potential the energy of the $2^{+}$resonant state in ${ }^{6} \mathrm{He}$ is $0.826 \mathrm{MeV}$ and its width is $0.075 \mathrm{MeV}$. To obtain converged (within a 5\% level) TF cross section we have included: (i) couplings to the $2^{+}$resonant state and nonresonant continuum (BU) states with partial waves for $\alpha-2 n$ relative motion up to f-waves ( $L=0,1,2$, and 3), (ii) the ${ }^{6} \mathrm{He}$ fragment-target potential multipoles up to the octopole term, and (iii) a maximum continuum energy of $8 \mathrm{MeV}$. All continuum couplings (including both bound-continuum

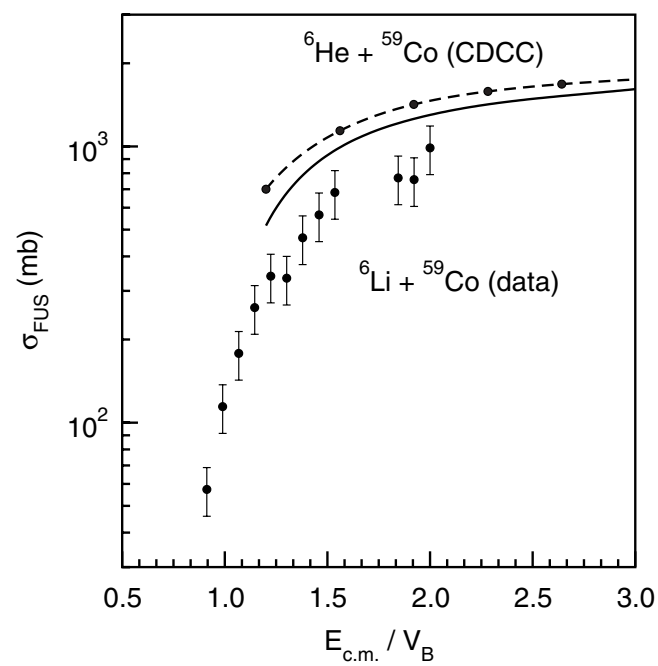

FIG. 8. Energy dependence of TF cross sections for the ${ }^{6} \mathrm{He}+{ }^{59} \mathrm{Co}$ reaction obtained with the CDCC method [29]. The dashed and thin curves correspond respectively to CDCC calculations with and without continuum couplings. The experimental TF cross sections for the ${ }^{6} \mathrm{Li}+{ }^{59} \mathrm{Co}$ system [44] are given for the sake of comparison. For each reaction, the incident energy is normalized by the Coulomb barrier of the effective potential $[69,70]$.

couplings and continuum-continuum couplings) were included in the calculation.

Results of the CDCC calculations for the TF fusion of ${ }^{6} \mathrm{He}+{ }^{59} \mathrm{Co}$ system are compared in two ways. First we present a comparison with the experimental excitation function of the ${ }^{6} \mathrm{Li}+{ }^{59} \mathrm{Co}$ system [44] as displayed in Fig. 8. An equivalent comparison with ${ }^{4} \mathrm{He}+{ }^{59} \mathrm{Co}$ data [71] is given in Fig. 9. Note that the calculation presented for the latter system is a simple two-body scattering calculation with an OM potential with an interior imaginary part simulating the ingoing wave boundary condition. In both cases we note that the bare no coupling

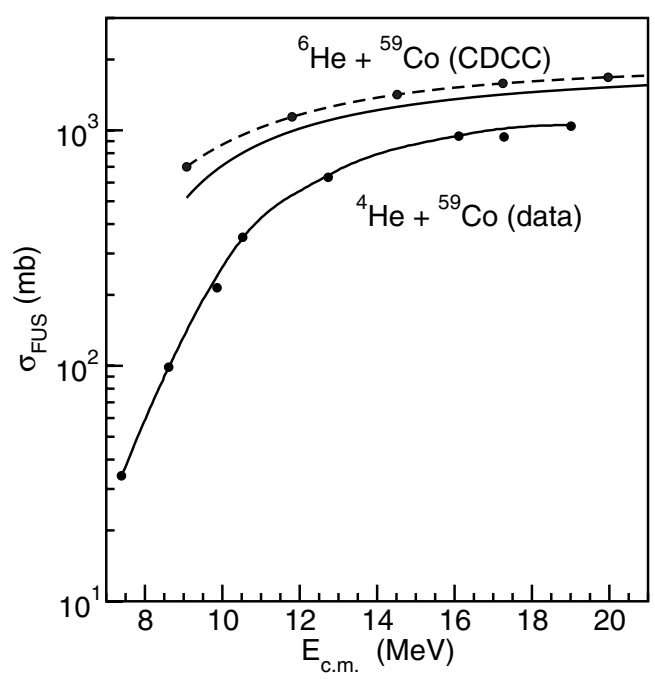

FIG. 9. The ${ }^{6} \mathrm{He}+{ }^{59} \mathrm{Co} \mathrm{TF}$ excitation functions are the same as in Fig. 8 and are compared with ${ }^{4} \mathrm{He}+{ }^{59} \mathrm{Co} \mathrm{TF}$ excitation functions. The TF cross sections of ${ }^{4} \mathrm{He}+{ }^{59} \mathrm{Co}$ were taken from [71] and standard calculations (solid curve) were performed as discussed in the text. 
TABLE V. TF cross sections obtained from the CDCC calculations for ${ }^{6} \mathrm{He}+{ }^{65} \mathrm{Cu}$ without $\left(\mathrm{th}_{1}\right)$ and with $\left(\mathrm{th}_{2}\right)$ continuum couplings are compared with measured total residue cross sections (exp) [16]. The total reaction cross sections extracted from $\mathrm{OM}$ fits to the elastic scattering data and deduced BU cross sections [16] are also given for comparison.

\begin{tabular}{lccccc}
\hline \hline $\begin{array}{l}E_{\mathrm{lab}} \\
(\mathrm{MeV})\end{array}$ & $\sigma_{t h_{1}}(\mathrm{mb})$ & $\sigma_{t h_{2}}(\mathrm{mb})$ & $\sigma_{\exp }(\mathrm{mb})$ & $\begin{array}{c}\sigma_{R}(\mathrm{OM}) \\
(\mathrm{mb})\end{array}$ & $\sigma_{\mathrm{b} u}(\mathrm{mb})$ \\
\hline 30 & 1637 & 1846 & 1334 & 1614 & 280 \\
19.5 & 1371 & 1606 & 1292 & 1502 & 210 \\
\hline \hline
\end{tabular}

TF calculation is already considerably larger than the TF cross sections for either ${ }^{6} \mathrm{Li}+{ }^{59} \mathrm{Co}$ or ${ }^{4} \mathrm{He}+{ }^{59} \mathrm{Co}$, and that the breakup couplings further increase the calculated TF cross sections at all energies investigated here.

Calculations were also performed for other medium-mass targets such as ${ }^{63,65} \mathrm{Cu}$ and ${ }^{64} \mathrm{Zn}$ nuclei. Their results are summarized for the copper isotopes in Tables V and VI along with experimental results reported in [16]. Here we again see that the effect of the breakup couplings is to increase the TF fusion cross section. However, the final values are rather larger than the data [16], of the order of 20-30\%. A similar conclusion is found for the zinc target at both near-barrier and sub-barrier energies [15]. This discrepancy could be due to the real potentials used (particularly when used for the ${ }^{2} n+$ target potentials) given that the bare no coupling values for the TF are already slightly larger than the measured ones or may be indicative of other coupling effects; coupling to single neutron stripping has been found to significantly reduce the TF cross section for ${ }^{6} \mathrm{He}$ at similar energies with respect to the Coulomb barrier [7].

\section{DISCUSSION}

It has already been remarked that there is some confusion about the definition of fusion $[6,29]$. Theorists usually define complete fusion (CF) as the capture of all the projectile fragments, and incomplete fusion (ICF) as the capture of only some fragments [29]. As in all other CC calculations, CDCC has the disadvantage of being unable to distinguish between CF and ICF. The combined effect of BU and TR in the CC approach has not been studied so far in the context of sub-barrier fusion. Another complication in experiments arises from a clear separation of CF and ICF cross sections,

TABLE VI. TF cross sections obtained from the CDCC calculations for ${ }^{6} \mathrm{He}+{ }^{63} \mathrm{Cu}$ without $\left(\mathrm{th}_{1}\right)$ and with $\left(\mathrm{th}_{2}\right)$ continuum couplings are compared with total residue cross sections (exp) measured at $30 \mathrm{MeV}$ [16]. The total reaction cross sections and BU cross sections were not reported in [16].

\begin{tabular}{lccccc}
\hline \hline $\begin{array}{l}E_{\mathrm{lab}} \\
(\mathrm{MeV})\end{array}$ & $\sigma_{t h_{1}}(\mathrm{mb})$ & $\sigma_{t h_{2}}(\mathrm{mb})$ & $\sigma_{\mathrm{exp}}(\mathrm{mb})$ & $\begin{array}{c}\sigma_{R}(\mathrm{OM}) \\
(\mathrm{mb})\end{array}$ & $\sigma_{\mathrm{b} u}(\mathrm{mb})$ \\
\hline 30 & 1600 & 1830 & 1400 & - & - \\
19.5 & 1349 & 1585 & - & - & - \\
\hline \hline
\end{tabular}

therefore $\mathrm{CF}$ is often defined experimentally as the capture of all the charge of the projectile by the target [72,73], although this definition would lead to problems for neutron halo nuclei such as ${ }^{6} \mathrm{He}$. In the following we discuss only TF cross sections (the sum of CF and ICF cross sections).

The standard three-body CDCC model is adequate for ${ }^{6,7} \mathrm{Li}$ as core excitation may be safely ignored for an $\alpha$ particle core. The elastic scattering data [48] as plotted in Figs. 1 and 2 are found to be very well reproduced for both the ${ }^{7} \mathrm{Li}$ and ${ }^{6} \mathrm{Li}$ nuclei, at least for the three highest incident energies. It is clear that despite the essentially negligible contribution of BU to the total reaction cross section coupling to BU has an important effect on the elastic scattering for both systems. Although the total reaction cross sections are dominated by $\mathrm{TF}$, it is also clear that the sum of $\mathrm{TF}+\mathrm{BU}$ by no means exhausts the total reaction cross section. As target excitation is expected to be relatively weak for ${ }^{59} \mathrm{Co}$ this leaves TR reactions as the other main contributor to the total reaction cross section, see, e.g., [57] and [74,75] for medium-mass and light targets, respectively. The effect of TR coupling on elastic scattering for weakly bound light projectiles remains to be fully elucidated, although it could be important depending on the system, see, e.g., $[76,77]$. It has already been demonstrated that the form of CDCC adapted to TF calculations is able to well describe TF for the ${ }^{6,7} \mathrm{Li}+{ }^{59} \mathrm{Co}$ systems [29].

Less clear is the question of whether either system exhibits a TA. Within the uncertainties, the surface strengths of the real parts of the best fit OM potentials show no dependence on incident energy for either isotope. This is also true for the imaginary part for ${ }^{7} \mathrm{Li}$, while the imaginary part for ${ }^{6} \mathrm{Li}$ seems to show a gradual rise in surface strength as the incident energy is reduced toward threshold, as seen previously for other targets $[78,79]$. However, given the somewhat artificial constraints employed in the grid searches carried out to define the error bars on the OM potentials one may equally argue that the ${ }^{6} \mathrm{Li}$ imaginary potential surface strength is also consistent with little or no variation with incident energy.

An overview of the available elastic scattering data for lithium isotopes from a variety of targets: ${ }^{208} \mathrm{~Pb}$ [78], ${ }^{138} \mathrm{Ba}$ [80], ${ }^{28} \mathrm{Si}$ [81], and ${ }^{27} \mathrm{Al}[82,83]$ does not allow any firm general conclusions concerning the presence or absence of the TA for either isotope. Part of the problem lies in the need for high precision data if one is to reduce the ambiguities in the extracted $\mathrm{OM}$ potential surface strengths to a level where firm conclusions as to their dependence on incident energy may be drawn. This is particularly true for the region around the "Coulomb rainbow" for the real part of the potential. There is also the question of dependence on target mass; there is no a priori reason to suppose that the TA found to be present in the ${ }^{7} \mathrm{Li}+{ }^{208} \mathrm{~Pb}$ system [78] will necessarily also be present in a system with a lighter target. For the ${ }^{7} \mathrm{Li}+{ }^{208} \mathrm{~Pb}$ system it was shown that coupling to the ${ }^{208} \mathrm{~Pb}\left({ }^{7} \mathrm{Li},{ }^{6} \mathrm{Li}\right){ }^{209} \mathrm{~Pb}$ transfer, with a negative reaction $\mathrm{Q}$-value, could account for the presence of TA [76]. However, for a ${ }^{58} \mathrm{Ni}$ target the reaction Q-value for the same stripping reaction is now positive, and it has been found that TR reactions with positive Q-values can give rise to DPPs that have similar properties to those produced by BU couplings [77]. From the present analysis with the ${ }^{59} \mathrm{Co}$ medium-mass target, it still remains unclear how the BU 
coupling affects the TA present for all tightly bound nuclei and if the concept of BU threshold anomaly [83,84] will be needed.

With no data available for ${ }^{6} \mathrm{He}+{ }^{59} \mathrm{Co}$ we cautiously decide not to present CDCC calculations for the elastic scattering for this system as the Coulomb dipole excitation is known to be too strong in the dineutron approximation [34], although a similar core-dineutron model [85] is capable of describing reasonably well the main properties of ${ }^{6} \mathrm{He}$; e.g., the nuclear charge radius, which measurements recently reported with high precision [86], was well predicted (to within 5\%). The dipole Coulomb excitation of ${ }^{6} \mathrm{He}$ projectiles in the field of a highly charged target has already been discussed [28,30,32,34]. The dineutron CDCC model has been found to give much better agreement with elastic scattering data when the dipole coupling strength is reduced by $50 \%$ [34]. This reduction is important for heavy targets, but probably not as much for a medium-mass target like ${ }^{59} \mathrm{Co}$. Nevertheless, such a reduction also reduces the total absorption cross section in the CDCC calculations. If we consider this cross section as approximating to the TF cross section, we may overestimate the fusion of ${ }^{6} \mathrm{He}+{ }^{59} \mathrm{Co}$ slightly.

The CDCC calculations for the ${ }^{6} \mathrm{He}+{ }^{59} \mathrm{Co} \mathrm{TF}$ described in the previous section are displayed in Figs. 8 and 9. They do not include either target excitations or TR channels. However, crude estimations such as those performed for the ${ }^{6} \mathrm{Li}+{ }^{59} \mathrm{Co}$ reaction [29] find the effect of target excitation to be very small. In Fig. 8 we compare the TF excitation functions for ${ }^{6} \mathrm{He}+{ }^{59} \mathrm{Co}$ (CDCC calculations) and ${ }^{6} \mathrm{Li}+{ }^{59} \mathrm{Co}$ (experimental data of [44]). We note that both calculated curves for ${ }^{6} \mathrm{He}$, with (dashed line) and without (solid line) BU couplings, give much larger TF cross sections than for ${ }^{6} \mathrm{Li}$. Similar conclusions are reached when the ${ }^{6} \mathrm{He}+{ }^{59} \mathrm{Co} \mathrm{TF}$ excitation function (CDCC calculations) is compared to that for ${ }^{4} \mathrm{He}+{ }^{59} \mathrm{Co}$ (here standard calculations fit the data of [71] remarkably well) in Fig. 9. This is a general result for medium-mass targets and does not depend on the nature of the target, as shown in Tables V and VI for two different copper isotopes. However, the calculations for ${ }^{6} \mathrm{He}+{ }^{63,65} \mathrm{Cu}$ somewhat over predict the measured TF cross sections. This could be due to the bare potentials used as input (the bare no coupling calculations give TF cross sections that are larger than the measured values, and the BU coupling consistently leads to an increase of the TF cross section in the CDCC model), the overestimation of the BU coupling effect on TF due to the use of the two-body dineutron model of ${ }^{6} \mathrm{He}$, or the effect of TR couplings, found to decrease the TF cross section for ${ }^{6} \mathrm{He}$ at similar incident energies with respect to the Coulomb barrier [7]. Unfortunately, this latter hypothesis cannot be tested in these systems due to the high density of states in the residual nuclei involved, ruling out a practicable calculation.

The present CDCC results, i.e., an increase in the TF cross section due to BU couplings, are in agreement with an alternate CC approach proposed by Dasso and Vitturi [87] that mimics continuum-continuum couplings in the BU channels. However, contradictory results have been obtained by Ito et al. [88] with a different approach based on a time-dependent wave-packet formalism suggesting the possible importance of higher partial waves for the relative motion between the valence particle and the projectile core. The converged cross sections within the CDCC approach (the study of the convergence of the results with respect to the number of angular momentum states in the continuum is discussed with great care in [29]) are found to be in reasonable agreement with the available TF data for medium-mass targets [16] (see Table III). This conclusion is consistent with similar CDCC calculations performed for heavy targets [28,31,34] and using the dineutron model. It should be mentioned that a recent study [38] of the ${ }^{6} \mathrm{He}+{ }^{209} \mathrm{Bi}$ reaction indicates that the $\alpha+n+n+{ }^{209} \mathrm{Bi}$ four-body model provides a more accurate description of the ${ }^{6} \mathrm{He}$ elastic scattering within the CDCC formalism than the dineutron model. It would be interesting to see what difference this more accurate model would have on the BU coupling effect on TF if applied to a fusion calculation in a similar manner to the calculations presented here.

\section{SUMMARY AND CONCLUDING REMARKS}

Halo and weakly bound cluster nuclei are good test-benches for theories of BU and fusion. We have shown that strong CC effects can be taken into account within a CDCC approach to model breakup effects on the angular distributions of the elastic scattering and on the excitation functions of the total $(\mathrm{CF}+$ ICF) fusion for reactions induced by ${ }^{6,7} \mathrm{Li}$ and ${ }^{6} \mathrm{He}$ projectiles. Although BU does not contribute significantly to the total reaction cross section at near-barrier energies, its influence is decisive for a fairly good description of the ${ }^{6,7} \mathrm{Li}+{ }^{59} \mathrm{Co}$ elastic scattering data. For both systems the total reaction cross sections are dominated by fusion at near and above barrier energies. The CDCC calculations suggest that there are other direct reaction processes (most likely nucleon TR) with larger contributions to the total reaction cross section than $\mathrm{BU}$. The real and imaginary parts of the ${ }^{6} \mathrm{Li}+{ }^{59} \mathrm{Co}$ and ${ }^{7} \mathrm{Li}+{ }^{59} \mathrm{Co}$ DPPs generated from the best $\mathrm{OM}$ fits to their respective elastic scattering angular distributions do not allow us to draw any concrete conclusions concerning the occurrence or not of the TA phenomenon in these systems.

Near-barrier TF cross sections calculated by CDCC for ${ }^{6} \mathrm{He}+{ }^{59} \mathrm{Co}$ are much larger than the measured TF yields for ${ }^{6} \mathrm{Li}+{ }^{59} \mathrm{Co}[44]$ and ${ }^{4} \mathrm{He}+{ }^{59} \mathrm{Co}[71]$ that are well reproduced by calculations. However, similar CDCC calculations for the ${ }^{6} \mathrm{He}+{ }^{63,65} \mathrm{Cu}$ systems somewhat over predict the data [16]. This may be due to deficiencies in the two-body model of ${ }^{6} \mathrm{He}$ used, to the global potentials used as a basis for the calculations or to the neglect of coupling to other reaction channels, e.g., TR. The present CDCC calculations show a consistent enhancement of the TF cross section due to coupling to $\mathrm{BU}$ with respect to the no coupling calculations. However, for a general description of fusion induced by ${ }^{6} \mathrm{He}$ projectiles a more complete theoretical model of few-body quantum dynamics that is able to (i) distinguish CF from ICF and (ii) treat explicitly TR channels is required and which will need to follow correlations after BU [26]. The two-body CDCC calculations [26-31,34] of the type we have presented in this work can serve as a good starting point; while ${ }^{6} \mathrm{He}$ is best described as a three-body $\alpha-n-n$ object, a two-body $\alpha-{ }^{2} n$ model appears to be satisfactory provided the $E_{1}$ strength is reduced by a factor of $\sim 0.5$ [34]. This is consistent with the fact that the mean charge radius measured for the two-neutron halo 
nucleus ${ }^{6} \mathrm{He}[86]$ can be fairly well described by dineutron cluster models [85].

The CDCC formalism, with continuum-continuum couplings taken into account, is probably one of the most reliable methods available nowadays to study reactions induced by weakly bound nuclei and exotic halo nuclei, although many of the latter have added complications like core excitation and three-body structure that are only now being incorporated within the formalism [35-40]. One really needs to investigate such processes within the dynamics of the interaction at the Coulomb barrier with loosely bound halo nuclei. An understanding of the reaction dynamics involving couplings to $\mathrm{BU}$ channels requires the explicit measurement of elastic scattering data with a high degree of precision as well as yields leading to the TR and BU channels. The complexity of such reactions, where many processes compete on an equal footing, necessitates kinematically and spectroscopically complete measurements, i.e., ones in which all processes from elastic scattering to fusion are measured simultaneously, providing a technical challenge in the design of broad range detection systems. A systematic study of ${ }^{6} \mathrm{He}$ induced fusion reactions with the CDCC method is still awaited, as up to now only very few experimental studies with ${ }^{6} \mathrm{He}$ projectiles are available $[4,10,12,14-16]$. A new experimental program with
SPIRAL beams and medium-mass targets is getting underway at GANIL.

The application of four-body CDCC models under current development [35-40] will then be highly desirable. The questions in the theory of a two-neutron halo system such as ${ }^{6} \mathrm{He}$, its $\mathrm{BU}$ (and in the breakup of many-body projectiles generally), and its CF and ICF will need knowledge not just of those integrated cross sections, but the phase space distributions of the surviving fragment(s). Therefore, future very exclusive experiments will need to determine very precisely the spatial (angular and energy) correlations of the individual neutrons. Preliminary attempts at measurements $[14,17]$ of $\alpha$-particles in coincidence with neutrons are promising.

\section{ACKNOWLEDGMENTS}

The authors would like to warmly thank F. A. Souza for supplying the elastic scattering cross sections of [48] in tabulated form. We also acknowledge P. R. S. Gomes, S. Kailas, R. Raabe, K. Rusek, and V. Zagrebaev for stimulating and instructive discussions. One of us (N.K.) gratefully acknowledges the receipt of a Marie Curie Intra-European grant from the European Commission, contract No. MEIFCT-2005-010158.
[1] A. B. Balantekin and N. Takigawa, Rev. Mod. Phys. 70, 77 (1998)

[2] M. Dasgupta, D. J. Hinde, N. Rowley, and A. M. Stefanini, Annu. Rev. Nucl. Part. Sci. 48, 401 (1998).

[3] K. Hagino, A. Vitturi, C. H. Dasso, and S. M. Lenzi, Phys. Rev. C 61, 037602 (2000).

[4] R. Raabe et al., Nature (London) 431, 823 (2004).

[5] J. F. Liang and C. Signorini, Int. J. Mod. Phys. E 14, 1121 (2006).

[6] L. F. Canto, P. R. S. Gomes, R. Donangelo, and M. S. Hussein, Phys. Rep. 424, 1 (2006).

[7] N. Keeley, R. Raabe, N. Alamanos, and J.-L. Sida, Progress in Particle and Nuclear Physics (in press); nucl-ex/0702038.

[8] C. Signorini et al., Nucl. Phys. A735, 329 (2004).

[9] V. I. Zagrebaev, Phys. Rev. C 67, 061601(R) (2003); private communication.

[10] Yu. E. Penionzhkevich, V. I. Zagrebaev, S. M. Lukyanov, and R. Kalpakchieva, Phys. Rev. Lett. 96, 162701 (2006).

[11] Yu. E. Penionzhkevich et al., Eur. Phys. J. A 31, 185 (2007); private communication.

[12] J. J. Kolata et al., Phys. Rev. Lett. 81, 4580 (1998).

[13] E. F. Aguilera et al., Phys. Rev. Lett. 84, 5058 (2000); Phys. Rev. C 63, 061603(R) (2001).

[14] J. P. Bychowski et al., Phys. Lett. B596, 62 (2004).

[15] A. Di Pietro et al., Phys. Rev. C 69, 044613 (2004).

[16] A. Navin et al., Phys. Rev. C 70, 044601 (2004).

[17] P. A. De Young et al., Phys. Rev. C 71, 051601(R) (2005); J. J. Kolata et al., ibid. 75, 031302(R) (2007); private communication.

[18] J. J. Kolata, Phys. Rev. C 71, 067603 (2005).

[19] C. H. Dasso, S. Landowne, and A. Winther, Nucl. Phys. A432, 495 (1985).
[20] G. H. Rawitscher, Phys. Rev. C 9, 2210 (1974); G. Rawitscher and I. Koltracht, J. Phys. G: Nucl. Part. Phys. 31, S1589 (2005) .

[21] M. Kamimura, M. Yahiro, Y. Iseri, Y. Sakuragi, H. Kameyama, and M. Kawai, Prog. Theor. Phys. (Kyoto) Suppl. 89, 1 (1986).

[22] M. Yahiro, Y. Iseri, H. Kameyama, M. Kamimura, and M. Kawai, Prog. Theor. Phys. (Kyoto) Suppl. 89, 32 (1986); M. Yahiro and M. Kamimura, Prog. Theor. Phys. (Kyoto) 65, 2046 (1981); 65, 2051 (1981).

[23] Y. Sakuragi, M. Yahiro, and M. Kamimura, Prog. Theor. Phys. (Kyoto) Suppl. 89, 136 (1986).

[24] N. Austern, Y. Iseri, M. Kamimura, M. Kawai, G. Rawitscher, and M. Yahiro, Phys. Rep. 154, 125 (1987), and references therein.

[25] J. A. Tostevin, F. M. Nunes, and I. J. Thompson, Phys. Rev. C 63, 024617 (2001).

[26] A. Diaz-Torres and I. J. Thompson, Phys. Rev. C 65, 024606 (2002).

[27] N. Keeley, K. W. Kemper, and K. Rusek, Phys. Rev. C 66, 044605 (2002).

[28] K. Rusek, N. Keeley, K. W. Kemper, and R. Raabe, Phys. Rev. C 67, 041604(R) (2003).

[29] A. Diaz-Torres, I. J. Thompson, and C. Beck, Phys. Rev. C 68 , 044607 (2003).

[30] N. Keeley, J. M. Cook, K. W. Kemper, B. T. Roeder, W. D. Weintraub, F. Maréchal, and K. Rusek, Phys. Rev. C 68, 054601 (2003).

[31] K. Rusek, N. Alamanos, N. Keeley, V. Lapoux, and A. Pakou, Phys. Rev. C 70, 014603 (2004).

[32] R. S. Mackintosh and N. Keeley, Phys. Rev. C 70, 024604 (2004).

[33] N. Keeley and R. S. Mackintosh, Phys. Rev. C 71, 057601 (2005). 
[34] K. Rusek, I. Martel, J. Gomez-Camacho, A. M. Moro, and R. Raabe, Phys. Rev. C 72, 037603 (2005).

[35] T. Matsumoto, E. Hiyama, M. Yahiro, K. Ogata, Y. Iseri, and M. Kamimura, Nucl. Phys. A738, 471 (2004).

[36] T. Matsumoto, E. Hiyama, K. Ogata, Y. Iseri, M. Kamimura, S. Chiba, and M. Yahiro, Phys. Rev. C 70, 061601(R) (2004).

[37] M. Rodriguez-Gallardo, J. M. Arias, J. Gomez-Camacho, A. M. Moro, I. J. Thompson, and J. A. Tostevin, Phys. Rev. C 72, 024007 (2005).

[38] T. Matsumoto, T. Egami, K. Ogata, Y. Iseri, M. Kamimura, and M. Yahiro, Phys. Rev. C 73, 051602(R) (2006)

[39] N. C. Summers, F. M. Nunes, and I. J. Thompson, Phys. Rev. C 73, 031603(R) (2006).

[40] N. C. Summers, F. M. Nunes, and I. J. Thompson, Phys. Rev. C 74, 014606 (2006).

[41] T. Aumann et al., Phys. Rev. C 59, 1252 (1999).

[42] C. Beck, A. Sànchez i Zafra, A. Diaz-Torres, I. J. Thompson, N. Keeley, and F. A. Souza, AIP Conf. Proc. No. 853 (AIP, New York, 2006), p. 354; arXiv:nucl-th/0605029.

[43] C. Beck, Nucl. Phys. A 787, 251c (2007).

[44] C. Beck, F. A. Souza, N. Rowley, S. J. Sanders, N. Aissaoui, E. E. Alonso, P. Bednarczyk, N. Carlin, S. Courtin, A. Diaz-Torres et al., Phys. Rev. C 67, 054602 (2003).

[45] F. A. Souza et al., Nucl. Phys. A718, 544c (2003).

[46] A. Szanto de Toledo et al., Nucl. Phys. A722, 248c (2004).

[47] A. Szanto de Toledo et al., Nucl. Phys. A734, 311c (2004).

[48] F. A. Souza et al., Phys. Rev. C 75, 044601 (2007); C. Beck et al., arXiv:nucl-ex/0411002.

[49] I. J. Thompson, Comput. Phys. Rep. 7, 167 (1988).

[50] K.-I. Kubo and M. Hirata, Nucl. Phys. A187, 186 (1972).

[51] B. Buck and A. C. Merchant, J. Phys. G 14, L211 (1988).

[52] F. Eigenbrod, Z. Phys. 228, 337 (1969).

[53] V. Avrigeanu, P. E. Hodgson, and M. Avrigeanu, Phys. Rev. C 49, 2136 (1994).

[54] L. McFadden and G. R. Satchler, Nucl. Phys. 84, 177 (1966).

[55] C. M. Perey and F. G. Perey, Phys. Rev. 132, 755 (1963).

[56] F. D. Becchetti, Jr., and G. W. Greenlees, in Polarization Phenomena in Nuclear Reactions edited by H. H. Barschall and W. Haeberli (The University of Wisconsin Press, Madison, WI, 1971), p. 682; quoted in C. M. Perey and F. G. Perey, At. Data Nucl. Data Tables 17, 1 (1976) .

[57] A. Shrivastava, A. Navin, N. Keeley, K. Mahata, K. Ramachandran, V. Nanal, V. V. Parkar, A. Chatterjee, and S. Kailas, Phys. Lett. B633, 463 (2006).

[58] C. B. Fulmer, G. R. Satchler, E. E. Gross, F. E. Bertrand, C. D. Goodman, D. C. Hensley, J. R. Wu, N. M. Clarke, and M. F. Steeden, Nucl. Phys. A356, 235 (1981).

[59] J. Cook, Nucl. Phys. A388, 153 (1982).
[60] O. V. Bochkarev, A. A. Korsheninnikov, E. A. Kuzmin, I. G. Mukha, L. V. Chulkov, and G. B. Yankov, Yad. Fiz. 41, 31 (1985); Sov. J. Nucl. Phys. 41(1), 19 (1985) .

[61] K. W. Kemper, A. F. Zeller, T. R. Ophel, D. F. Hebbard, A. Johnston, and D. C. Weisser, Nucl. Phys. A320, 413 (1979).

[62] I. J. Thompson, M. A. Nagarajan, J. S. Lilley, and M. J. Smithson, Nucl. Phys. A505, 84 (1989).

[63] S. Cohen and D. Kurath, Nucl. Phys. A101, 1 (1967).

[64] J. N. Roy, H. M. Sen Gupta, A. R. Majumder, T. Congedo, and J. E. Alzona, J. Phys. G 4, 1469 (1978).

[65] R. Ballini, A. G. Blair, N. Cindro, J. Delaunay, and J. P. Fouan, Nucl. Phys. A111, 147 (1968).

[66] R. G. H. Robertson and R. G. Summers-Gill, Can. J. Phys. 49, 1186 (1971).

[67] M. Niwano, T. Ishimatsu, R. Asano, T. Suehiro, and M. Tanaka, Nucl. Phys. A377, 148 (1982).

[68] M. H. MacFarlane and S. C. Pieper, Phys. Lett. 103B, 169 (1981).

[69] R. A. Broglia and A. Winther, Heavy-Ion Reactions, Parts I and II, Frontiers in Physics Lecture Notes Series, Vol. 84 (Addison-Wesley, New York, 1991).

[70] P. R. Christensen and A. Winther, Phys. Lett. B65, 19 (1976).

[71] M. A. McMahan and J. M. Alexander, Phys. Rev. C 21, 1261 (1980).

[72] M. Dasgupta et al., Phys. Rev. C 70, 024606 (2004).

[73] Z. H. Liu et al., Eur. Phys. J. A 26, 73 (2005).

[74] A. Pakou et al., Phys. Rev. Lett. 90, 202701 (2003).

[75] A. Pakou et al., Phys. Rev. C 69, 054602 (2004).

[76] N. Keeley and K. Rusek, Phys. Rev. C 56, 3421 (1997).

[77] N. Keeley, N. Alamanos, K. Rusek, and K. W. Kemper, Phys. Rev. C 71, 014611 (2005).

[78] N. Keeley, S. J. Bennett, N. M. Clarke, B. R. Fulton, G. Tungate, P. V. Drumm, M. A. Nagarajan, and J. S. Lilley, Nucl. Phys. A571, 326 (1994).

[79] A. Pakou et al., Phys. Lett. B556, 21 (2003).

[80] A. M. Maciel et al., Phys. Rev. C 59, 2103 (1999).

[81] A. Pakou et al., Phys. Rev. C 69, 054602 (2004).

[82] J. M. Figueira et al., Phys. Rev. C 73, 054603 (2006).

[83] J. M. Figueira et al., Phys. Rev. C 75, 017602 (2007).

[84] M. S. Hussein, P. R. S. Gomes, J. Lubian, and L. C. Chamon, Phys. Rev. C 73, 044610 (2006).

[85] H. Esbensen, G. F. Bertsch, and K. Hencken, Phys. Rev. C 56, 3054 (1997).

[86] L.-B. Wang et al., Phys. Rev. Lett. 93, 142501 (2004).

[87] C. H. Dasso and A. Vitturi, J. Phys. (London): Nucl. Part. Phys. G 31, S1449 (2005).

[88] M. Ito, K. Yabana, T. Nakatsukasa, and M. Ueda, Phys. Lett. B637, 53 (2006). 